\title{
OPEN A case-control study investigating food addiction in Parkinson patients
}

Ingrid de Chazeron ${ }^{1 凶}$, F. Durif ${ }^{2}$, C. Lambert ${ }^{3}$, I. Chereau-Boudet ${ }^{1}$, M. L. Fantini ${ }^{4}$, A. Marques ${ }^{2}$, P. Derost ${ }^{2}$, B. Debilly ${ }^{2}$, G. Brousse ${ }^{1}$, Y. Boirie ${ }^{5,6}$ \& P. M. Llorca ${ }^{1,2,3,4,5,6}$

Eating disorders (EDs) in patients with Parkinson's disease (PD) are mainly described through impulse control disorders but represent one end of the spectrum of food addiction (FA). Although not formally recognized by DSM-5, FA is well described in the literature on animal models and humans, but data on prevalence and risk factors compared with healthy controls (HCs) are lacking. We conducted a crosssectional study including 200 patients with PD and 200 age- and gender-matched HCs. Characteristics including clinical data (features of PD/current medication) were collected. FA was rated using DSM-5 criteria and the Questionnaire on Eating and Weight Patterns-Revised (QEWP-R). Patients with PD had more EDs compared to HCs $(27.0 \%$ vs. $13.0 \%$, respectively, $p<0.001)$. They mainly had FA $(24.5 \%$ vs. $12.0 \%, p=0.001)$ and night eating syndrome $(7.0 \%$ vs. $2.5 \% p=0.03)$. In PD patients, FA was associated with female gender $(p=0.04)$ and impulsivity (higher attentional non-planning factor) but not with the dose or class of dopaminergic therapy. Vigilance is necessary, especially for PD women and in patients with specific impulsive personality traits. Counterintuitively, agonist dopaminergic treatment should not be used as an indication for screening FA in patients with PD.

In Parkinson's disease (PD), compulsive eating is part of the spectrum of impulse control disorders (ICDs) that also include pathologic gambling, compulsive buying and hypersexuality ${ }^{1}$. Eating disorders (EDs) are abnormal eating habits that negatively affect a person's physical and/or mental health ${ }^{2}$. The psychiatric community categorizes these disorders as separate entities with a distinct set of symptoms [i.e. binge eating disorder (BED)] but the general term may also include food addiction (FA). The concept of FA, a highly controversial subject, refers to people who exhibit substance dependence symptoms in relation to certain high-fat and high-sugar foods ${ }^{3}$.

The prevalence of EDs (e.g. binge eating disorder or compulsive eating) in patients with PD varies greatly from 0.32 to $11.13 \% \%^{4,5}$. This wide range reflects the various methods (e.g., telephone interviews, patient selfreports, retrospective database research regarding psychological scales, etc.) and tools used, but it also reflects the variability of ED symptomatology. Compulsive eating is characterized by the uncontrollable consumption of a larger amount of food than usual, in excess of that necessary to alleviate hunger. We previously observed that the definition of EDs should include symptoms of food addiction (FA) corresponding to a diminution of control over consumption, which appears to be a core feature ${ }^{6}$. Furthermore, whether or not binge eating disorder (compulsive overeating, BED) points to an addiction remains unclear ${ }^{7}$.

In PD, a relationship between EDs and dopaminergic treatment, particularly dopamine agonists, has been mainly suspected, although the combined influence of various factors ${ }^{8}$ has been mentioned. As a result, it is necessary to identify the precise underlying neurobiological substrates ${ }^{9}$. Therefore, even if new animal models of ICDs are being implemented, it would also be of interest to investigate these behaviors in patients without PD at the same ages. However, little is known regarding the prevalence and symptomatology of FA in the general elderly population. We do not have data on FA or the age of disease onset for the general population. A recent cross-national community survey revealed a median age-of-onset in the late teens to early $20 \mathrm{~s}$ and a persistence of 4.3 years ${ }^{10}$ for a specific pathology such as BED. Like in many other investigations, the representation of subjects $\geq 60$ years old was weaker and could not correctly describe this phenomenon in this population.

\footnotetext{
${ }^{1}$ Service de Psychiatrie B, CHU Clermont-Ferrand, EA7280, Université Clermont Auvergne, Rue Montalembert BP 69, 63003 Clermont-Ferrand, France. ${ }^{2}$ Service de Neurologie A, CHU Clermont-Ferrand, EA7280, Université Clermont Auvergne, Clermont-Ferrand, France. ${ }^{3}$ Unité de Biostatistiques (DRCl), CHU Clermont-Ferrand, Clermont-Ferrand, France. " Explorations Fonctionnelles du Système Nerveux, CHU Clermont-Ferrand, EA7280, Université Clermont Auvergne, Clermont-Ferrand, France. ${ }^{5}$ Service de Nutrition Clinique, INRA, UMR 1019, UNH, CRNH Auvergne, CHU Clermont-Ferrand, Clermont-Ferrand, France. ${ }^{6}$ Centre Référent Troubles Des Conduites Alimentaires, CHU Clermont-Ferrand, Clermont-Ferrand, France. ${ }^{\circledR}$ email: idechazeron@chu-clermontferrand.fr
} 
Previous work suggests that BED is particularly prevalent in older adults, and the prevalence of all eating disorders was around $3.5 \%$ in older women and $1-2 \%$ in older men ${ }^{11,12}$. Indeed, people with EDs can experience varied symptoms, ranging from BED to bulimia nervosa or other specific feeding and eating disorders (OSFED) that have been included in recent modifications of DSM- $5^{2}$ in order to better represent their behaviors and symptoms. These behaviors and symptoms can also be embedded in FA. The new nosological entity of FA ${ }^{13}$ could be of interest when investigating EDs in patients with PD. FA is a clinical and multidimensional concept. Gearhardt et al. ${ }^{14}$ proposed the concept, confirmed by Gordon et al. ${ }^{3}$, of FA as a specific phenotype defined by applying the diagnostic criteria for substance dependence to particular processed foods with added sweeteners or fats. FA was found to be more prevalent not only in obese individuals ${ }^{15}$ but also in patients with alterations in brain circuitry similar to those found in drug addiction ${ }^{16}$.

Some studies have already investigated the relationship between FA and BED such as their overlaps and possible unique differences, although this has generally been done in specific populations like obese participants ${ }^{17}$.

The aim of this study was to explore the prevalence, clinical spectrum and personality profile correlates of FA in a group of subjects with PD compared with age- and gender-matched healthy controls (HCs).

\section{Methods}

Participants. Patients with PD (based on the UK Brain Bank criteria) were screened during regularly scheduled follow-up visits at the Movement Disorders Unit of Clermont-Ferrand Hospital, France, over a 12-month period. HCs were recruited at health check centers in the same region over a 24-month period.

Exclusion criteria were chronic illness (with the exception of PD for the PD group and no significant neurological dysfunction for the HC group) that could influence eating habits (e.g., diabetes) and inability to understand the instructions (language or cognitive impairment based on the Mini-Mental State Examination <24). HCs were matched (1:1) for age ( \pm 4 years) and gender and all had no prior history of a neurological disorder. Initially, 220 subjects with PD and $230 \mathrm{HCs}$ were recruited, 20 were excluded in the PD group and 30 in the HC group. Subjects were treated under routine clinical care. The STROBE guidelines ${ }^{18}$ were used to ensure the reporting of this paired study. All the methods used for this study were carried out in accordance with relevant guidelines and regulations. All the procedures performed in the studies involving human participants were in accordance with the ethical standards of the institutional and/or national research committee. The study was approved by the French Ethic Committee. It has been qualified as an observational study and need of informed consent was waived by this Committees of Protection of Persons South-East 6 pursuant to L.1121-1-1 and R.1121-3 of the French Public Health Code in force at the time of the research.

Study outcomes. Sociodemographic and anthropometric parameters (height and weight) and clinical data (features of PD), including medical details, were collected. On the same day, patients with PD underwent the following assessment procedures: PD medical history and current medication were collected through patient interviews and confirmed with medical records. Anti-Parkinson medications were converted into a levodopa equivalent daily dose (LEDD) ${ }^{19}$. Subjects were then administered sections of the Movement Disorders Society Unified Parkinson Disease Rating Scale (MDS-UPDRS), which includes an interview and examination by a movement disorder neurologist. Subscores for non-motor experiences of daily living (part I) and motor examination (part III) of the MDS-UPDRS were calculated.

We assessed neuropsychiatric symptoms, such as punding, compulsive buying, pathological gambling and hypersexuality using questions derived from the standardized interview "Ardouin Scale of Behavior in Parkinson's Disease" (ASBPD) ${ }^{20}$.

We assessed psychological distress with the Hospital Anxiety and Depression Scale (HADS) in patients with $\mathrm{PD}$ and $\mathrm{HCs}^{21}$. HADS is a self-assessment questionnaire that has been found to be a reliable instrument for detecting states of anxiety and depression; seven items are related to depression and seven to anxiety subscales. Scoring for each item ranges from zero to three, with three denoting the highest anxiety or depression level. A total subscale score of $>11$ points out of a possible 21 denotes considerable symptoms of anxiety or depression.

Impulsivity was assessed with the Barratt impulsiveness scale (BIS-11) ${ }^{22}$, which is a 30 -item questionnaire designed to assess common impulsive behaviors in three separable dimensions: attentional impulsiveness (defined as the [in-]ability to concentrate or focus attention), motor impulsiveness (tendency to act without thinking), and non-planning impulsiveness (lack of future planning and forethought). BIS-11 is composed of 30 items scored on a Likert scale (ranging from never $=1$ point to very frequently $=4$ points). The total score is the sum of all the items and it also assesses the three main dimensions of impulsive behavior: attentional (11 items), motor (11 items), and non-planning impulsivity (8 items).

Following the interview and examination, the participants were asked to provide individual information regarding food habits and EDs, including FA (DSM-5 criteria and the Questionnaire on Eating and Weight Patterns-Revised [QEWP-R] $)^{23}$ and food cravings ${ }^{24}$.

The food craving questionnaire is composed of 14 questions used in a general evaluation study ${ }^{24}$. Three items scored on a Likert scale (ranging from 0 to 4 points rated proportionally to frequency or intensity). One item measures alteration of their daily rhythm of life to obtain a particular food (binary data). We used it only for the description of food craving in relation to a dimension already well known in other specific questionnaires like the FCQ (intense desire to eat...).

The QEWP-R primarily focuses on assessing diagnostic criteria for BED but also assesses night eating syndrome (NES) and includes clinically significant impairment or distress, criteria needed for FA diagnosis. QEWP$\mathrm{R}$ is a 28-item self-report instrument. It is designed to assess the components, duration, and frequency requirements for BED diagnosis. 
The Yale Food Addiction Scale (YFAS) ${ }^{25}$ is currently the best available measure for evaluating food addiction, but a variety of approaches have also been used to measure FA including self-report questionnaires ${ }^{26}$ and patient self-identification ${ }^{27}$. This study was conducted during a routine regular visit. We usually use psychiatric and neurological assessments in the ward but were unable to introduce a new tool, so we decided to use only 2 items from the DSM-5 criteria for FA symptoms: 1/Food often consumed in larger amounts or over a longer period or more frequently than was intended, and 2/Craving, or a strong desire or urge to eat specific foods. These items have already been empirically supported in the concept of $\mathrm{FA}^{28}$. FA is diagnosed when the participant reports two symptoms (DSM-5) plus clinically significant impairment/distress (QEWP-R). This scoring is in accordance with YFAS2.0 $0^{29}$.

NES is a circadian delay in the pattern of daily food intake, with evening hyperphagia and/or nocturnal ingestions of food. The term EDs in this paper is used to include FA and more characterized eating troubles, such as BED, NES, purging bulimia nervosa, non-purging bulimia nervosa, etc. The quality of food that induces craving has been classified by the FDA and Drewnowski ${ }^{30}$ into two categories regarding sugar-fat ratio.

Statistical methods. Statistical analysis was performed using Stata software (StataCorp. 2015. Stata Statistical Software: Release 14. College Station, TX: StataCorp LP). The tests were two-sided, with a type I error set at $\alpha=0.05$. Categorical parameters were expressed as frequencies and associated percentages, and continuous data as means and standard deviation (SDs) or as medians and interquartile ranges (IQRs), according to statistical distribution. The prevalence of FA was presented with a $95 \%$ confidence interval (CI). Categorical variables were compared between independent groups (PD vs. HC or subjects with vs. without FA) using the chi-squared test or Fisher's exact test. Quantitative data were compared between groups with Student's t-test or with the Mann-Whitney test, as appropriate. The Gaussian distribution was verified by the Shapiro-Wilk test, and homoscedasticity was verified by the Fisher-Snedecor test. In the context of multivariate analysis, generalized linear mixed models with the logit link function were used, considering the pair (because each patient with PD was matched with an HC) as a random effect. The dependent variable of the models was binary (FA yes or no), and the independent variables were selected according to univariate results and clinical relevance. The results were expressed as odds ratios (ORs) and 95\% CIs.

\section{Results}

There were 200 subjects with PD and 200 HCs. The distribution by age and gender was similar between groups due to the matched design. There were $60 \%$ men $(n=120)$ and the mean age was 67.5 years old (SD 9.9). The characteristics of patients with PD and HCs are presented in Table 1. There was no difference between groups for occupational situation, even before being retired. No between-group difference was observed for body mass index (BMI), even looking separately by gender subgroup, but there were significantly more patients with PD with an underweight BMI class compared to HCs and significantly more HCs in in the obesity BMI class than PD subjects. The mean age at onset of PD was 60.2 years (SD 10.0), and the median disease duration was 5.8 years (IQR: 3.0; 10.7). The mean MDS-UPDRS for the medication score for parts I (non-motor experiences of daily living) and III (motor examination) were 1.7 (SD 1.8) and 16.7 (SD 9.8), respectively. Four patients with PD received no dopaminergic treatment, and 173 received levodopa (LEDD $583 \mathrm{mg} /$ day, IQR: 350; 910) either as monotherapy (54 cases) or in association with other medications ( $49.1 \%$ received dopamine agonist [DA] medication). Regarding the last 23 PD patients (LEDD $232 \mathrm{mg}$ /day, IQR: 100; 500), 4 had DA alone, 7 monoamine oxidase inhibitors (MAO-I) and 12 an association of DA and MAO-I.

More cases of compulsive buying were detected in the PD population compared to the HCs ( $8.5 \%$ vs. $2.5 \%$, respectively, $p=0.008$ ), but there were no differences for punding, pathological gambling and hypersexuality. Based on the suggested HADS cut-off values, $29.0 \%$ and $15.0 \%$ of patients with PD had definite anxiety and depression cases, significantly more than in the HC population $(p<0.05)$. Patients with PD also had a significantly higher HADS-depression score compared to HCs (6.6 [SD 3.9] vs. 4.5 [SD 3.5], respectively, $p<0.001$ ). Overall, patients with PD showed a mean BIS-11 total score and non-planning impulsivity score significantly higher than those in HCs (respectively $p=0.02$ and $p<0.001$ ), although these differences stayed minimal.

PD vs HCs subjects. Compared to HCs (Table 2), significantly more subjects with PD had changed their food habits $(28.0 \%$ vs. $18.0 \% p=0.02)$, especially in the last 5 years, which corresponded to their disease duration. Indeed, a large majority (89.3\%) of patients related their changes in food habits to PD diagnosis. Compared to HCs, subjects with PD ate mostly between meals (51.0\% vs. $36.0 \%$ ), $12.5 \%$ of PD subjects ate between meals during night and day. There were significantly two-fold more EDs in the population with PD compared to HCs $(27.0 \%$ vs. $13.0 \%, p<0.001)$. EDs were largely represented by FA cases (plus one case of purging bulimia nervosa and two cases of BED) (prevalence of FA in patients with PD: $24.5 \%$ [95\% CI 18.7, 31.1] vs. HCs $12.0 \%$ [95\% CI 7.8, 17.4]). NES was significantly more frequent in the PD group compared to the HC group (7.0\% vs. $2.5 \%$, $p=0.03)$. Regarding food craving, there were many symptomatologic differences between cases of ED in patients with PD or HCs, including frequency of intense desire to eat and strength of desire or difficulty to resist, which were more frequent in the PD group. All cases of EDs that were able to change their daily rhythm of life to satisfy a particular food craving were patients with PD. The food composition that induced craving was different between the PD and HC groups. Patients with PD preferred a high-sugar diet $(81.6 \%$ for patients with PD vs. $55.2 \%$ for HCs, $p=0.01$ ) rather than a high-fat diet (71.4\% for patients with PD vs. $79.3 \%$ for HCs, $p=0.44)$. Chocolate was largely considered as a craving food for $13.0 \%$ of subjects with PD vs. $4.5 \%$ for HCs $(p=0.003)$.

FA characteristics. We explored the characteristics of FA among the whole population of the study (subjects with PD and HCs) (Table 3). Compared to those without FA, there were significantly more female subjects 


\begin{tabular}{|c|c|c|c|}
\hline & HCs $(n=200)$ & PD $(n=200)$ & $p$ \\
\hline Occupational situation, $\mathrm{n}(\%)$ & & & 0.42 \\
\hline Working & $31(15.5)$ & $24(12.0)$ & \\
\hline Retired & $156(78.0)$ & $160(80.0)$ & \\
\hline Disability & $10(5.0)$ & $15(7.5)$ & \\
\hline Looking for a job & $3(1.5)$ & $1(0.5)$ & \\
\hline Occupational class when working, $\mathbf{n}(\%)$ & & & 0.10 \\
\hline Craft trade and firm managers & $1 / 31(3.2)$ & $4 / 24(16.7)$ & \\
\hline Upper managerial staff and professionals & $7 / 31(22.6)$ & $5 / 24(20.8)$ & \\
\hline Intermediary occupations & $5 / 31(16.1)$ & $0 / 24(0.0)$ & \\
\hline Clerks and employees & $18 / 31(58.1)$ & $15 / 24(62.5)$ & \\
\hline BMI $\left(\mathrm{kg} / \mathrm{m}^{2}\right)$, mean $(\mathrm{SD})$ & $25.9(4.2)$ & $25.1(4.1)$ & 0.07 \\
\hline Range, n (\%) & & & 0.04 \\
\hline$<18.5$ (Underweight) & $1(0.5)$ & $10(5.0)$ & \\
\hline 18.5 to $<25.0$ (Normal weight) & $90(45.0)$ & $91(45.5)$ & \\
\hline 25.0 to $<30.0$ (Overweight) & $83(41.5)$ & $80(40.0)$ & \\
\hline$\geq 30.0$ (Obesity) & $26(13.0)$ & $19(9.5)$ & \\
\hline Age at PD onset (years), mean (SD) & - & $60.2(10.0)$ & - \\
\hline Disease duration (years), median [IQR] & - & $5.8[3.0 ; 10.7]$ & - \\
\hline \multicolumn{4}{|l|}{ MDS-UPDRS on medication, mean (SD) } \\
\hline Part I & - & $1.7(1.8)$ & - \\
\hline Part III & - & $16.7(9.8)$ & - \\
\hline \multicolumn{4}{|l|}{ PD medication } \\
\hline Cumulative LEDD (mg/day), median [IQR] & - & $500[257 ; 850]$ & - \\
\hline Levodopa medication alone, $\mathrm{n}(\%)$ & - & $54(27.0)$ & - \\
\hline Levodopa dose (mg/day), median [IQR] & - & $375[83 ; 600]$ & - \\
\hline DA medication, n (\%) & - & $101(50.5)$ & - \\
\hline DA dose (LEDD, mg/day), median [IQR] & - & $35[0 ; 210]$ & - \\
\hline Punding, n (\%) & $62(31.0)$ & $54(27.0)$ & 0.38 \\
\hline Compulsive buying, n (\%) & $5(2.5)$ & $17(8.5)$ & 0.008 \\
\hline Pathological gambling, n (\%) & $2(1.0)$ & $7(3.5)$ & 0.18 \\
\hline Hypersexuality, n (\%) & $3(1.5)$ & $7(3.5)$ & 0.20 \\
\hline \multicolumn{4}{|l|}{ HADS } \\
\hline HADS-anxiety score, mean (SD) & $7.6(3.8)$ & $8.3(4.1)$ & 0.11 \\
\hline Anxiety cases, n (\%) & $41(20.5)$ & $58(29.0)$ & 0.049 \\
\hline HADS-depression score, mean (SD) & $4.5(3.5)$ & $6.6(3.9)$ & $<0.001$ \\
\hline Depression cases, n (\%) & $12(6.0)$ & $30(15.0)$ & 0.003 \\
\hline \multicolumn{4}{|l|}{ BIS-11, mean (SD) } \\
\hline Total score & $55.0(8.5)$ & $57.1(9.4)$ & 0.02 \\
\hline Motor factor & $17.1(3.3)$ & $17.2(3.8)$ & 0.64 \\
\hline Non-planning factor & $22.0(4.2)$ & $23.5(4.6)$ & $<0.001$ \\
\hline Attentional factor & $16.0(3.1)$ & $16.3(3.6)$ & 0.30 \\
\hline
\end{tabular}

Table 1. Description of HCs and patients with PD $(n=400)$. Bold values indicate statistical significance $(p<0.05)$. BMI body mass index, BIS-11 Barratt impulsiveness scale, DA medication dopamine agonist medication, HADS hospital anxiety and depression scale, HCs healthy controls, IQR interquartile range, $L E D D$ levodopa equivalent daily dose, $M A O-I$ monoamine oxidase inhibitor, $M D S$-UPDRS movement disorder society-unified Parkinson's disease rating scale, $P D$ Parkinson's disease, $S D$ standard deviation.

with FA $(53.4 \%$ vs. $37.0 \%, p=0.01)$, with a higher frequency of $\mathrm{PD}(67.1 \%$ vs. $46.2 \%, p=0.001)$. The FA group had a higher cumulative LEDD (372 mg/day IQR [0;663] vs. 0 [0; 450], $p=0.002)$, a higher levodopa dose (74 mg/day IQR $[0 ; 500]$ vs. 0 [0; 300], $p=0.008)$, more frequent DA medication $(38.4 \%$ vs. $22.3 \%, p=0.004)$ and a higher DA dose ( $0 \mathrm{mg} /$ day IQR $[0 ; 160]$ vs. $0[0 ; 0], p=0.007)$. They were more frequently compulsive buyers $(12.3 \%$ vs. $4.0 \%, p=0.009)$, more anxious (HADS-anxiety cases $37.0 \%$ vs. $22.0 \%, p=0.007)$ but not more depressed. They did not have a higher total impulsivity score (BIS-11), but when looking at the subscore, they had greater attentional impulsiveness $(p=0.006)$. Food composition (high-sugar or high-fat food) inducing food craving was not specific for the FA group compared to the no-FA group, except for chocolate. When looking at the characteristics of PD patients with and without FA, cumulative $\operatorname{LEDD}(583 \mathrm{mg} /$ day IQR $[372 ; 755]$ vs. 500 [241; 850], $p=0.47$ ), levodopa dose ( $375 \mathrm{mg} /$ day IQR [74; 750] vs. 365 [83; 600], $p=0.56$ ), presence of DA 


\begin{tabular}{|c|c|c|c|}
\hline & HCs $(n=200)$ & PD $(n=200)$ & $p$ \\
\hline Have changes in food habits, $\mathbf{n}(\%)$ & $36(18.0)$ & $56(28.0)$ & 0.02 \\
\hline Time for changes in food habits, $\mathbf{n}(\%)$ & & & 0.03 \\
\hline Never change & $164(82.0)$ & $144(72.0)$ & $0.05^{\dagger}$ \\
\hline Change in the last 5 years & $15(7.5)$ & $33(16.5)$ & $0.02^{\dagger}$ \\
\hline Change between 5 and 10 years & $15(7.5)$ & $19(9.5)$ & $0.52^{\dagger}$ \\
\hline Change more than a decade ago & $6(3.0)$ & $4(2.0)$ & $0.52^{\dagger}$ \\
\hline \multicolumn{4}{|l|}{ PD link and changes in food habits, $n(\%)$} \\
\hline Before PD diagnosis & - & $6 / 56(10.7)$ & - \\
\hline After PD diagnosis & - & $50 / 56(89.3)$ & - \\
\hline After PD treatment & - & $13 / 56(23.2)$ & - \\
\hline Actual food regularly consumed, $\mathrm{n}(\%)$ & & & $<0.001$ \\
\hline During the meal & $128(64.0)$ & $98(49.0)$ & $0.006^{\dagger}$ \\
\hline Between meals, during the day & $66(33.0)$ & $70(35.0)$ & $0.67^{\dagger}$ \\
\hline Between meals, during the night & $3(1.5)$ & $7(3.5)$ & $0.40^{\dagger}$ \\
\hline Between meals, night and day & $3(1.5)$ & $25(12.5)$ & $\mathbf{0 . 0 0 4}^{\dagger}$ \\
\hline EDs, n (\%) & $26(13.0)$ & $54(27.0)$ & $<0.001$ \\
\hline Purging bulimia nervosa, $\mathbf{n}(\%)$ & $0(0.0)$ & $1(0.5)$ & 1.00 \\
\hline Non-purging bulimia nervosa, $\mathbf{n}(\%)$ & $0(0.0)$ & $0(0.0)$ & - \\
\hline BED, n (\%) & $0(0.0)$ & $2(1.0)$ & 0.50 \\
\hline FA ( $\varnothing$ BED), $\mathbf{n}(\%)$ & $24(12.0)$ & $49(24.5)$ & 0.001 \\
\hline NES, $n(\%)$ & $5(2.5)$ & $14(7.0)$ & 0.03 \\
\hline \multicolumn{4}{|l|}{ Food craving, $n(\%)$} \\
\hline A strong/intense desire to eat more than once a week & $22(11.0)$ & $47(23.5)$ & 0.001 \\
\hline Strength of desire: mild to very strong & $24(12.0)$ & $53(26.5)$ & $<0.001$ \\
\hline Difficulty to resist the desire to eat: hard to impossible to resist & $17(8.5)$ & $39(19.5)$ & 0.002 \\
\hline $\begin{array}{l}\text { When they do not have their particular food, capable of altering their daily rhythm of life to get } \\
\text { it (i.e., go out to buy it at a store) }\end{array}$ & $0(0.0)$ & $8(4.0)$ & 0.007 \\
\hline \multicolumn{4}{|l|}{ Food composition inducing craving, $n(\%)$} \\
\hline High-sugar & \begin{tabular}{|l|}
$16 / 29(55.2)$ \\
\end{tabular} & \begin{tabular}{|l|}
$40 / 49(81.6)$ \\
\end{tabular} & 0.01 \\
\hline High-fat & $23 / 29(79.3)$ & $35 / 49(71.4)$ & 0.44 \\
\hline Chocolate & $9(4.5)$ & $26(13.0)$ & 0.003 \\
\hline
\end{tabular}

Table 2. Eating disorders in the HC and PD groups $(n=400)$. Bold values indicate statistical significance $(p<0.05)$. BED binge eating disorder, $F A$ food addiction, $H C$ healthy controls, $N E S$ night eating syndrome, $P D$ Parkinson's disease, EDs eating disorders, $\varnothing$ : without. ${ }^{\dagger}$ Post-hoc comparison.

medication (79.6\% vs. 70.9\%, $p=0.23)$, DA dose (210 mg/day IQR [129; 225] vs. 200 [120; 300], $p=0.2)$ were not significantly different between PD with or without FA. Compared to no-FA, FA-PD patients were more frequently compulsive buyers ( $16.3 \%$ vs. $6.0 \%, p=0.024)$, had a similar non-planning impulsiveness score $(p=0.87)$ but greater attentional impulsiveness $(p=0.024)$.

Multivariate analysis. The multivariate analysis was run with significant and clinically relevant factors from the univariate analysis on FA (Fig. 1A). This analysis showed that being a patient with PD (OR 2.40, 95\% CI $1.34 ; 4.29$ ) or a female (OR $1.89,95 \%$ CI 1.07; 3.33) significantly increases the risk of having an FA.

In particular, regarding the population with $\mathrm{PD}$ and FA, multivariate analysis (Fig. 1B) showed that being a female (OR 2.11, 95\% CI 1.05; 4.24) or having a high attentional impulsiveness score (OR 1.16, 95\% CI 1.04; 1.29 ) increases the risk of having an FA. A high non-planning impulsiveness score (OR 0.90, 95\% CI 0.83; 0.98) was protective. The use of DA treatment or being a compulsive buyer did not significantly influence the chances of having an FA in a patient with PD (the DA dose was not significant either, data not shown).

\section{Discussion}

In the present study, we investigated EDs in patients with PD compared to a matching general population. Our main findings were: (1) patients with PD had more EDs than the general population; (2) patients with PD had FA and NES, with marginal BED or bulimia; and (3) FA were related to gender and an impulsivity-specific dimension but not to treatment.

To our knowledge, this is the first study specifically investigating EDs in PD compared to a matched paired population with PD. Several studies have consistently shown the presence of EDs in patients with PD, but they generally observed only an extreme fringe of EDs that corresponded to BED and/or bulimia cases. A recent metaanalytic study ${ }^{31}$ on the risk of ICDs in patients with PD versus controls observed an OR from 0.66 to 17.05 for binge eating. These estimates were obtained with heterogeneous study designs, sample sizes, diagnostic criteria 


\begin{tabular}{|c|c|c|c|}
\hline & Without FA $(\mathbf{n}=327)$ & With FA $(n=73)$ & $p$ \\
\hline Gender, n (\% male) & $206(63.0)$ & $34(46.6)$ & 0.01 \\
\hline Age (years), mean (SD) & $67.9(9.8)$ & $65.8(10.1)$ & 0.10 \\
\hline BMI $\left(\mathrm{kg} / \mathrm{m}^{2}\right)$, mean $(\mathrm{SD})$ & $25.6(4.2)$ & $25.2(3.8)$ & 0.48 \\
\hline PD diagnosis, $n(\%)$ & $151(46.2)$ & $49(67.1)$ & 0.001 \\
\hline Cumulative LEDD (mg/day), median [IQR] & $0[0 ; 450]$ & $372[0 ; 663]$ & 0.002 \\
\hline Levodopa dose (mg/day), median [IQR] & $0[0 ; 300]$ & $74[0 ; 500]$ & 0.008 \\
\hline DA medication, n (\%) & $73(22.3)$ & $28(38.4)$ & 0.004 \\
\hline DA dose (LEDD, mg/day), median [IQR] & $0[0 ; 0]$ & $0[0 ; 160]$ & 0.007 \\
\hline Punding, n (\%) & $92(28.1)$ & $24(32.9)$ & 0.42 \\
\hline Compulsive buying, n (\%) & $13(4.0)$ & $9(12.3)$ & 0.009 \\
\hline Pathological gambling, n (\%) & $6(1.8)$ & $3(4.1)$ & 0.22 \\
\hline Hypersexuality, n (\%) & $8(2.4)$ & $2(2.7)$ & 1.00 \\
\hline \multicolumn{4}{|l|}{ HADS } \\
\hline HADS-anxiety score, mean (SD) & $7.7(4.0)$ & $8.9(3.7)$ & 0.02 \\
\hline Anxiety cases, n (\%) & $72(22.0)$ & $27(37.0)$ & 0.007 \\
\hline HADS-depression score, mean (SD) & $5.6(3.8)$ & $5.5(3.8)$ & 0.79 \\
\hline Depression cases, n (\%) & $34(10.4)$ & $8(11.0)$ & 0.89 \\
\hline \multicolumn{4}{|l|}{ BIS-11, mean (SD) } \\
\hline Total & $55.7(8.9)$ & $57.7(9.4)$ & 0.09 \\
\hline Motor & $17.1(3.4)$ & $17.5(4.0)$ & 0.46 \\
\hline Non-planning & $22.7(4.5)$ & $23.1(4.2)$ & 0.45 \\
\hline Attentional & $16.0(3.3)$ & $17.2(3.6)$ & 0.006 \\
\hline \multicolumn{4}{|l|}{ Food composition inducing craving, $\mathbf{n}(\%)$} \\
\hline High-sugar & $12 / 16(75.0)$ & $44 / 62(71.0)$ & 1.00 \\
\hline High-fat & $9 / 16(56.2)$ & $49 / 62(79.0)$ & 0.11 \\
\hline Chocolate & $7(2.1)$ & $28(38.4)$ & $<0.001$ \\
\hline
\end{tabular}

Table 3. Characteristics of food addiction (FA) subjects $(n=400)$. Bold values indicate statistical significance $(p<0.05)$. BIS-11 Barratt impulsiveness scale, BMI body mass index, DA medication dopamine agonist medication, HADS hospital anxiety and depression scale, $L E D D$ levodopa equivalent daily dose, PD Parkinson's disease, $S D$ standard deviation.

and ascertainment methods. In the literature, EDs (e.g. excessive drive to eat or binge-eating disorder) were described in up to $15 \%$ of patients with PD who were treated with a DA, leading to weight gain and sometimes to obesity ${ }^{32,33}$.

FA and NES in PD subjects. Our study is the first to show that FA is very common in patients with PD (24.5\%), far exceeding the rate of the general population $(9 \%)^{34}$. However, other alterations of eating behavior in the population with PD have been already described as "insatiable craving" "with uncontrollable traits" but they have not yet been estimated ${ }^{35-38}$. Based on recent systematic reviews by Pursey et al. ${ }^{15}$ and Burrows et al. ${ }^{39}$ it is estimated that FA affects approximately $20 \%$ of the population (range 5.4-56.8\%) but the great majority of studies have been performed outside Europe and concern specific populations such as patients attending bariatric surgery or obese subjects.

In our study, $7.0 \%$ of patients with PD had NES, which seems to be close to the observed prevalence of nocturnal eating in a prospective study ${ }^{40}$. However, in that study the patients observed underwent deep brain stimulation and therefore were at a more advanced stage of disease compared to our disease cohort, which corresponded to a symptomatic population with PD that had not reached an advanced stage. NES and sleeprelated eating disorders have been frequently reported in patients with idiopathic restless legs syndrome (RLS). Interestingly, PD patients may suffer from RLS and an increased frequency of NES was recently reported in PD with RLS compared to those without ${ }^{41}$. Unfortunately, RLS was not assessed in the present study, and we do not know whether the prevalence of RLS differed between PD patients and HCs. Further studies on ED in PD should take into account this potential association.

FA in the elderly and women. Few studies have focused on FA in the general population, especially in the elderly. One study ${ }^{42}$ focused exclusively on 342 women over the age of 65 and, according to the DSM- 5 criteria, observed a prevalence of all EDs at 3.25\%, with $1.68 \%$ being BED. Binge eating episodes were reported by $5.6 \%$ of women and picking/nibbling by $18.9 \%$ of women. These figures are close to ours, although our population was mixed in terms of gender and our FA criteria were absent from their study. Around $1.5 \%$ of individuals in the general population can present $\mathrm{NES}^{43}$. This syndrome has been more specifically observed in women, and $9.1 \%$ of them aged $\geq 55$ years old reported getting up during the night to eat in the study by Andersen ${ }^{44}$. Food cravings 
a

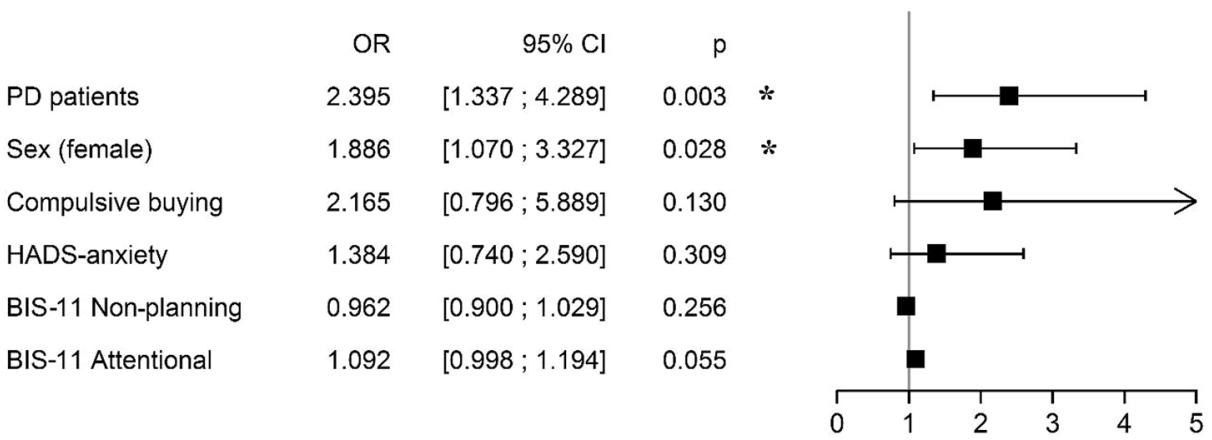

b

Sex (female)
DA medication
Compulsive buying
BIS-11 Non-planning

BIS-11 Attentional
OR

2.106

1.270

2.310

0.900

1.157
$95 \% \mathrm{Cl}$

[1.046;4.240]

[0.642; 2.512]

$[0.780 ; 6.844]$

$[0.828 ; 0.979]$

$[1.040 ; 1.288]$

$p$
$0.037 *$
0.492
0.131
$0.014 \quad *$
$0.007 \quad *$

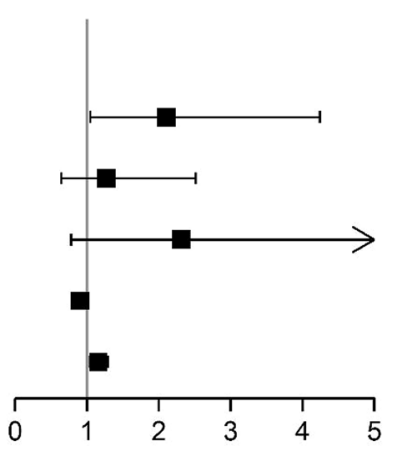

Figure 1. Multivariate analysis of food addiction (FA): $a$ in the whole population $(n=400)$ and $b$ in PD population $(\mathrm{n}=200)$. BIS-11 Non-planning Barratt impulsiveness scale subscore non-planning, BIS-11 Attentional Barratt impulsiveness scale subscore attentional, CI confidence interval, DA medication dopamine agonist medication, HADS-anxiety hospital anxiety and depression scale subscore anxiety, $O R$ odds ratio, $P D$ Parkinson's disease.

are extremely common, particularly among women ${ }^{45}$, which seems to be confirmed by our study when looking at the multivariate analysis results for FA for the whole population.

Cravings for specific types of foods, such as sweet or high-fat foods, has been described for women with $\mathrm{BED}^{46}$, but we did not observe this in the FA analyses, except for chocolate. Patients with PD also reported frequent cravings not only for chocolate but also for sugar-rich foods.

Association with ICDs and impulsivity. Overall, patients with PD showed more cases of compulsive buying, they had higher depression and anxiety scores and had depression and anxiety disorders more often compared to the general population, characteristics often described in the literature ${ }^{1,47}$ and still significant when comparing a population with or without FA. However, this specific ICD disappeared in the multivariate analysis, may be because it was more related to the over-representation of the PD group. Regarding the other associated disorders, we observed a close to significantly higher attentional impulsiveness score in the FA group, and this factor was still significant in the multivariate analysis in the subgroup of the population with PD (OR 1.16). Attentional impulsiveness has been defined as "an inability to focus attention or concentrate", and supports the association between FA and attentional deficits that has already been observed in individuals with obesity ${ }^{48,49}$. This factor was not significantly different between patients with PD and the general population, implying that it was very specific for FA. More surprisingly, non-planning impulsivity (orientation to the present rather than to the future) is a protective factor $(\mathrm{OR}=0.90)$ only for FA in patients with PD. Only one study ${ }^{50}$ observed a potential weakly positive relation between non-planning impulsivity and rigid control of eating behavior. The mean non-planning impulsivity score in patients with PD corresponded to normative values, and it is perhaps not directly related to an "eating decision" but rather "having access to food". Patients with PD who were able to reduce their non-planning impulsiveness (contrary to increasing their ability to plan) may be better able to buy food for later and then consume it when feeling the urge to eat.

Factors linked to FA in a pooled population. The investigation of factors that were linked to FA in a pooled population showed that a diagnosis of PD (OR 2.40) and being a woman (OR 1.89) are two major risk factors for FA. Gender specificity has been a constant finding in past research on FA in the general population ${ }^{15}$, while the risk factors for PD have been poorly studied ${ }^{6}$ because they have been mainly explored through the prism of the ICD spectrum when discussed around EDs. Kistner et al. ${ }^{51}$ proposed that the vast majority of EDs in patients with PD (excluding BED) should be interpreted as subthreshold pathological behaviors in order to compensate for low dopaminergic signaling and called it "hypodopaminergic snacking". In the present study, no difference was found between PD patients with or without FA in dopamine ligand type and dose, although dopa- 
mine is a well-known contributor to addiction through its differentiated roles in reinforcement, motivation and self-regulation ${ }^{52-54}$. Interestingly, studies of post-STN stimulation observed that the proportion of patients presenting excessive eating behavior remained high in the follow-up, suggesting that the underlying mechanisms of this specific behavior are complex and need forms of management other than just decreased medication ${ }^{51,55,56}$. On the other hand, we observed that impulsive personality modifications were related to FA. Previous studies have linked impulsive personality changes with alterations in dopaminergic activity and receptor availability ${ }^{57,58}$.

Limitations. A limitation of the study is that, given the context of recruitment, we did not use a specific scale for FA or Food craving. This may prevent this study from being directly comparable to previous research. In addition, actual dietary intake was not measured. Self-report measures were used. As a result, this tended to overestimate the prevalence rates ${ }^{59}$, but we used validated self-questionnaires. It would be interesting in future works to include rigorous control of food intakes and use specific scales like YFAS ${ }^{34}$. Moreover, we used questions to assess neuropsychiatric symptoms derived from the ASBPD, which has been validated only in populations with PD. The senses of smell and taste, which are known to influence eating behaviors and can be modified in $\mathrm{PD}^{60}$, were not explored in our study and future research could explore how these issues could affect eating behaviors in patients with PD. We could not explore whether or not dopamine treatment is a major risk factor for FA in the PD population because of the specificity of patients included (reference center for patients with advanced disease).

Finally, as multifactorial analyses were used, there was no way to determine causal relationships between significant factors and FA.

Although it seems that being attentive to food addiction in Parkinson's is not common, it should not be neglected, especially in women. New insights into the understanding of the mechanism of FA remain to be investigated, more specifically in patients with PD, and certain components of impulsivity might be targeted in future intervention programs.

Received: 20 July 2020; Accepted: 18 March 2021

Published online: 25 May 2021

\section{References}

1. Weintraub, D., David, A. S., Evans, A. H., Grant, J. E. \& Stacy, M. Clinical spectrum of impulse control disorders in Parkinson's disease. Mov. Disord. 30, 121-127. https://doi.org/10.1002/mds.26016 (2015).

2. American Psychiatric Association. Fifth Edition of the Diagnostic and Statistical Manual of Mental Disorders (DSM-5) (New School Library, 2013).

3. Gordon, E. L., Ariel-Donges, A. H., Bauman, V. \& Merlo, L. J. What is the evidence for "food addiction?" A systematic review. Nutrients https://doi.org/10.3390/nu10040477 (2018).

4. Cossu, G., Rinaldi, R. \& Colosimo, C. The rise and fall of impulse control behavior disorders. Parkinsonism Relat. Disord. 46(Suppl 1), S24-S29. https://doi.org/10.1016/j.parkreldis.2017.07.030 (2018).

5. Zahodne, L. B. et al. Binge eating in Parkinson's disease: prevalence, correlates and the contribution of deep brain stimulation. J. Neuropsychiatry Clin. Neurosci. 23, 56-62. https://doi.org/10.1176/appi.neuropsych.23.1.56 (2011).

6. de Chazeron, I. et al. Compulsive eating behaviors in Parkinson's disease. Eat. Weight Disord. 4, 019-00648 (2019).

7. Corwin, R. L. The face of uncertainty eats. Curr. Drug Abuse Rev. 4, 174-181 (2011).

8. Grall-Bronnec, M. et al. Dopamine agonists and impulse control disorders: a complex association. Drug Saf. 41, 19-75. https:// doi.org/10.1007/s40264-017-0590-6 (2018).

9. Napier, T. C. et al. Linking neuroscience with modern concepts of impulse control disorders in Parkinson's disease. Mov. Disord. 30, 141-149. https://doi.org/10.1002/mds.26068 (2015).

10. Kessler, R. C. et al. The prevalence and correlates of binge eating disorder in the World Health Organization world mental health surveys. Biol. Psychiatry 73, 904-914. https://doi.org/10.1016/j.biopsych.2012.11.020 (2013).

11. Mangweth-Matzek, B. \& Hoek, H. W. Epidemiology and treatment of eating disorders in men and women of middle and older age. Curr. Opin. Psychiatry 30, 446-451. https://doi.org/10.1097/yco.0000000000000356 (2017).

12. Lapid, M. I. et al. Eating disorders in the elderly. Int. Psychogeriatr. 22, 523-536. https://doi.org/10.1017/s1041610210000104 (2010).

13. Fletcher, P. C. \& Kenny, P. J. Food addiction: A valid concept?. Neuropsychopharmacology 43, 2506-2513. https://doi.org/10.1038/ s41386-018-0203-9 (2018).

14. Gearhardt, A. N., Corbin, W. R. \& Brownell, K. D. Food addiction: an examination of the diagnostic criteria for dependence. J. Addict. Med. 3, 1-7. https://doi.org/10.1097/ADM.0b013e318193c993 (2009).

15. Pursey, K. M., Stanwell, P., Gearhardt, A. N., Collins, C. E. \& Burrows, T. L. The prevalence of food addiction as assessed by the yale food addiction scale: a systematic review. Nutrients 6, 4552-4590. https://doi.org/10.3390/nu6104552 (2014).

16. Gearhardt, A. N. et al. Neural correlates of food addiction. Arch. Gen. Psychiatry 68, 808-816. https://doi.org/10.1001/archgenpsy chiatry.2011.32 (2011).

17. Burrows, T., Skinner, J., McKenna, R. \& Rollo, M. Food addiction, binge eating disorder, and obesity: Is there a relationship?. Behav. Sci. (Basel) https://doi.org/10.3390/bs7030054 (2017).

18. von Elm, E. et al. The strengthening the reporting of observational studies in epidemiology (STROBE) statement: guidelines for reporting observational studies. PLoS Med. https://doi.org/10.1371/journal.pmed.0040296 (2007).

19. Tomlinson, C. L. et al. Systematic review of levodopa dose equivalency reporting in Parkinson's disease. Mov. Disord. 25, 2649-2653. https://doi.org/10.1002/mds.23429 (2010).

20. Rieu, I. et al. International validation of a behavioral scale in Parkinson's disease without dementia. Mov. Disord. 30, 705-713. https://doi.org/10.1002/mds.26223 (2015).

21. Zigmond, A. S. \& Snaith, R. P. The hospital anxiety and depression scale. Acta Psychiatr. Scand. 67, 361-370 (1983).

22. Patton, J. H., Stanford, M. S. \& Barratt, E. S. Factor structure of the Barratt impulsiveness scale. J. Clin. Psychol. 51, 768-774 (1995).

23. Yanovski, S. Z. Binge eating disorder: current knowledge and future directions. Obes. Res. 1, 306-324 (1993).

24. Guy-Grand, B., Pouillon, M. \& Le Barzic, M. Caractérisation des "accés alimentaires impulsifs" chez les sujets obéses". Cahiers Nutr. Diététique 32, 307-312 (1997).

25. Gearhardt, A. N., Corbin, W. R. \& Brownell, K. D. Preliminary validation of the yale food addiction scale. Appetite 52, 430-436. https://doi.org/10.1016/j.appet.2008.12.003 (2009). 
26. Merlo, L. J., Klingman, C., Malasanos, T. H. \& Silverstein, J. H. Exploration of food addiction in pediatric patients: a preliminary investigation. J. Addict. Med. 3, 26-32. https://doi.org/10.1097/ADM.0b013e31819638b0 (2009).

27. Ifland, J. R. et al. Refined food addiction: a classic substance use disorder. Med. Hypotheses 72, 518-526. https://doi.org/10.1016/j. mehy.2008.11.035 (2009).

28. Meule, A. \& Gearhardt, A. N. Food addiction in the light of DSM-5. Nutrients 6, 3653-3671. https://doi.org/10.3390/nu6093653 (2014).

29. Gearhardt, A. N., Corbin, W. R. \& Brownell, K. D. Development of the yale food addiction scale version 2.0. Psychol. Addict. Behav. 30, 113-121. https://doi.org/10.1037/adb0000136 (2016).

30. Drewnowski, A. Metabolic determinants of binge eating. Addict. Behav. 20, 733-745. https://doi.org/10.1016/0306-4603(1095) 00105-00100 (1995).

31. Molde, H. et al. Impulse-control disorders in Parkinson's disease: a meta-analysis and review of case-control studies. Front. Neurol. 9, 330. https://doi.org/10.3389/fneur.2018.00330 (2018).

32. Giladi, N., Weitzman, N., Schreiber, S., Shabtai, H. \& Peretz, C. New onset heightened interest or drive for gambling, shopping, eating or sexual activity in patients with Parkinson's disease: the role of dopamine agonist treatment and age at motor symptoms onset. J. Psychopharmacol. 21, 501-506 (2007).

33. Weintraub, D. et al. Impulse control disorders in Parkinson disease: a cross-sectional study of 3090 patients. Arch. Neurol. 67, 589-595. https://doi.org/10.1001/archneurol.2010.65 (2010).

34. Brunault, P., Ballon, N., Gaillard, P., Réveillère, C. \& Courtois, R. Validation of the French version of the yale food addiction scale: an examination of its factor structure, reliability, and construct validity in a nonclinical sample. Can. J. Psychiatry 59, 276-284. https://doi.org/10.1177/070674371405900507 (2014).

35. Hinnell, C., Hulse, N., Martin, A. \& Samuel, M. Hypersexuality and compulsive over-eating associated with transdermal dopamine agonist therapy. Parkinsonism Relat. Disord. 17, 295-296. https://doi.org/10.1016/j.parkreldis.2011.01.010 (2011).

36. Khan, W. \& Rana, A. Q. Dopamine agonist induced compulsive eating behaviour in a Parkinson's disease patient. Pharm. World Sci. 32, 114-116. https://doi.org/10.1007/s11096-009-9358-0 (2010).

37. Nirenberg, M. J. \& Waters, C. Compulsive eating and weight gain related to dopamine agonist use. Mov. Disord. 21, 524-529 (2006).

38. Vitale, C. et al. Comparative neuropsychological profile of pathological gambling, hypersexuality, and compulsive eating in Parkinson's disease. Mov. Disord. 26, 830-836. https://doi.org/10.1002/mds.23567 (2011).

39. Burrows, T., Kay-Lambkin, F., Pursey, K., Skinner, J. \& Dayas, C. Food addiction and associations with mental health symptoms: a systematic review with meta-analysis. J. Hum. Nutr. Diet 31, 544-572. https://doi.org/10.1111/jhn.12532 (2018).

40. Lhommée, E. et al. Subthalamic stimulation in Parkinson's disease: restoring the balance of motivated behaviours. Brain 135, 1463-1477. https://doi.org/10.1093/brain/aws078 (2012).

41. Marques, A. et al. Impulse control disorders in Parkinson's disease patients with RLS: a cross sectional-study. Sleep Med. 48, 148-154. https://doi.org/10.1016/j.sleep.2018.02.004 (2018).

42. Conceicao, E. M., Gomes, F. V. S., Vaz, A. R., Pinto-Bastos, A. \& Machado, P. P. P. Prevalence of eating disorders and picking/ nibbling in elderly women. Int. J. Eat. Disord. 50, 793-800. https://doi.org/10.1002/eat.22700 (2017).

43. Rand, C. S., Macgregor, A. M. \& Stunkard, A. J. The night eating syndrome in the general population and among postoperative obesity surgery patients. Int. J. Eat. Disord. 22, 65-69. https://doi.org/10.1002/(sici)1098-108x(199707)22:1\%3c65::aid-eat8\%3e3.0. co;2-0 (1997).

44. Andersen, G. S., Stunkard, A. J., Sørensen, T. I., Petersen, L. \& Heitmann, B. L. Night eating and weight change in middle-aged men and women. Int. J. Obes. Relat. Metab. Disord. 28, 1338-1343. https://doi.org/10.1038/sj.ijo.0802731 (2004).

45. Weingarten, H. P. \& Elston, D. The phenomenology of food cravings. Appetite 15, 231-246 (1990).

46. Yanovski, S. Sugar and fat: cravings and aversions. J. Nutr. 133, 835S-837S. https://doi.org/10.1093/jn/133.3.835S (2003).

47. Schrag, A. \& Taddei, R. N. Depression and anxiety in Parkinson's disease. Int. Rev. Neurobiol. 133, 623-655. https://doi.org/10. 1016/bs.irn.2017.05.024 (2017).

48. Meule, A., de Zwaan, M. \& Müller, A. Attentional and motor impulsivity interactively predict "food addiction" in obese individuals. Compr. Psychiatry 72, 83-87. https://doi.org/10.1016/j.comppsych.2016.10.001 (2017).

49. Steward, T. et al. Food addiction and impaired executive functions in women with obesity. Eur. Eat. Disord. Rev. 26, 574-584. https://doi.org/10.1002/erv.2636 (2018).

50. Timko, C. A. \& Perone, J. Rigid and flexible control of eating behavior in a college population. Eat. Behav. 6, 119-125. https://doi. org/10.1016/j.eatbeh.2004.09.002 (2005).

51. Kistner, A., Lhommee, E. \& Krack, P. Mechanisms of body weight fluctuations in Parkinson's disease. Front. Neurol. 5, 84. https:// doi.org/10.3389/fneur.2014.00084 (2014).

52. Fernagut, P. O. Dopamine and eating behavior disorders in Parkinson's disease: a complex recipe. Mov. Disord. 34, 767-768. https:// doi.org/10.1002/mds.27703 (2019)

53. Ho, A. L. et al. Deep brain stimulation for obesity: rationale and approach to trial design. Neurosurg. Focus 38, E8. https://doi.org/ 10.3171/2015.3.focus1538 (2015).

54. Volkow, N. D., Wise, R. A. \& Baler, R. The dopamine motive system: implications for drug and food addiction. Nat. Rev. Neurosci. 18, 741-752. https://doi.org/10.1038/nrn.2017.130 (2017).

55. Eusebio, A. et al. Subthalamic nucleus stimulation and compulsive use of dopaminergic medication in Parkinson's disease. J. Neurol. Neurosurg. Psychiatry 84, 868-874. https://doi.org/10.1136/jnnp-2012-302387 (2013).

56. Castrioto, A., Lhommee, E., Moro, E. \& Krack, P. Mood and behavioural effects of subthalamic stimulation in Parkinson's disease. Lancet Neurol. 13, 287-305. https://doi.org/10.1016/s1474-4422(13)70294-1 (2014).

57. Caravaggio, F. et al. Exploring personality traits related to dopamine D2/3 receptor availability in striatal subregions of humans. Eur. Neuropsychopharmacol. 26, 644-652. https://doi.org/10.1016/j.euroneuro.2016.02.010 (2016).

58. Weiland, B. J. et al. Relationship between impulsivity, prefrontal anticipatory activation, and striatal dopamine release during rewarded task performance. Psychiatry Res. 223, 244-252. https://doi.org/10.1016/j.pscychresns.2014.05.015 (2014).

59. Celio, A. A., Wilfley, D. E., Crow, S. J., Mitchell, J. \& Walsh, B. T. A comparison of the binge eating scale, questionnaire for eating and weight patterns-revised, and eating disorder examination questionnaire with instructions with the eating disorder examination in the assessment of binge eating disorder and its symptoms. Int. J. Eat. Disord. 36, 434-444 (2004).

60. Bannier, S. et al. Prevalence and phenomenology of olfactory hallucinations in Parkinson's disease. J. Neurol. Neurosurg. Psychiatry 83, 1019-1021. https://doi.org/10.1136/jnnp-2012-302414 (2012).

\section{Acknowledgements}

The project was supported by the Hospital Program for Clinical Research (local funding) at Clermont-Ferrand University Hospital-FRANCE and TEVA Santé France. We wish to acknowledge the Association ISBA Health Prevention ('Association ISBA Santé Prévention') for their support with data for the HCs. 


\section{Author contributions}

d.C.I.: Research project: conception, organization; statistical analysis: design, review and critique; manuscript: writing of the first draft and corrections. D.F.: Research project: conception, execution; manuscript: review and critique. L.C.: statistical analysis: design, execution; manuscript: review and critique. C.-B.I.: Research project: conception. F.L.: Research project: execution. M.A.: research project: execution. D.P.: research project: execution. D.B.: research project: execution. B.G.: manuscript: review and critique. B.Y.: manuscript: review and critique. L.P.M.: research project: conception; statistical analysis: review and critique; manuscript: review and critique.

\section{Funding}

Full financial disclosure for the previous 12 months: All authors that received financial or non-financial support received it outside the framework of the work submitted. de Chazeron I.: None. Durif F.: Non-financial support from AbbVie laboratory and Sanofi France. Lambert C.: None. Chereau-Boudet I.: Non-financial support from Janssen-Cilag France Laboratory. Fantini L.: None. Marques A.: Non-financial support from Merz, UCB Pharma SA, LivaNova SAS, Aguettant Laboratory, Ever Pharma France, Biogen France, Abbot medical France. Derost P.: Non-financial support from LVL Medical Groupe. Debilly B.: Non-financial support from LVL Medical Groupe and Biogen France. Brousse G.: Non-financial support from Camurus AB, Laboratory Bouchara-Recordati. Boirie Y.: Non-financial support from Nutrica Nutrition Clinique France and Fresenius Kabi France. Llorca P.M.: Expert groups: Allergan, Eisai, Gedeon Richter, Janssen, Lundbeck, Otsuka, Recordatti, Sanofi, Teva/Coordination of clinical studies: Janssen/Financing of research projects, fees, financing of travel and accommodation at conferences, consultant, speaker at symposia: Allergan, Eisai, Gedeon Richter, Janssen, Lundbeck, Otsuka, Recordatti, Sanofi, Teva. The study was supported by a regional public grant (AOI CHU Clermont-Ferrand 2010) and partially supported by a grant from TEVA Santé France. The funder had no role in the writing of the study protocol or in carrying out the study.

\section{Competing interests}

The authors declare no competing interests.

\section{Additional information}

Correspondence and requests for materials should be addressed to I.C.

Reprints and permissions information is available at www.nature.com/reprints.

Publisher's note Springer Nature remains neutral with regard to jurisdictional claims in published maps and institutional affiliations.

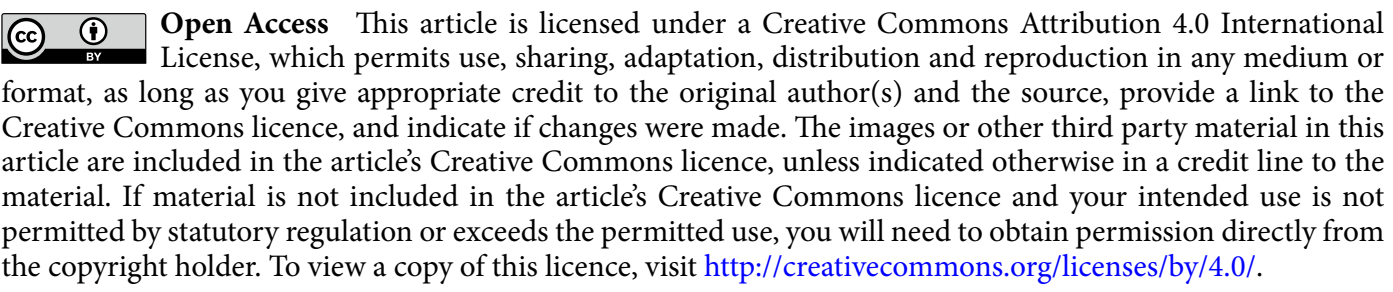

(C) The Author(s) 2021 